2013

\title{
Alpha(1)-adrenergic-mediated eNOS phosphorylation in intact arteries
}

Robin C. Looft-Wilson

William \& Mary, rlooft@wm.edu

Sarah E. Todd

William \& Mary

Christina A. Araj

William \& Mary

Stephanie M. Mutchler

William \& Mary

Cara A. Raphael Goodell

William \& Mary

Follow this and additional works at: https://scholarworks.wm.edu/aspubs

\section{Recommended Citation}

Looft-Wilson, R. C., Todd, S. E., Araj, C. A., Mutchler, S. M., \& Goodell, C. A. R. (2013). Alpha1-adrenergicmediated eNOS phosphorylation in intact arteries. Vascular pharmacology, 58(1-2), 112-117.

This Article is brought to you for free and open access by the Arts and Sciences at W\&M ScholarWorks. It has been accepted for inclusion in Arts \& Sciences Articles by an authorized administrator of W\&M ScholarWorks. For more information, please contact scholarworks@wm.edu. 


\title{
Alpha $_{1}$-adrenergic-mediated eNOS phosphorylation in intact arteries
}

\author{
Robin C. Looft-Wilson *, Sarah E. Todd, Christina A. Araj, Stephanie M. Mutchler, Cara A. Raphael Goodell \\ Department of Kinesiology and Health Sciences, The College of William E Mary, P.0. Box 8795, Williamsburg, VA 23187, United States
}

\section{A R T I C L E I N F O}

\section{Article history:}

Received 25 May 2012

Received in revised form 23 August 2012

Accepted 4 September 2012

\section{Keywords:}

Mouse

Mesenteric

Myoendothelial junctions

Vasodilation

Phenylephrine

Acetylcholine

\begin{abstract}
A B S T R A C T
Activation of arterial smooth muscle alpha ${ }_{1}$-adrenergic receptors results in vasoconstriction, as well as a secondary release of nitric oxide and slow vasodilation, presumably through gap junction communication from smooth muscle to endothelium. We hypothesized that this slow vasodilation is due to activation of eNOS through phosphorylation at Ser1179 and dephosphorylation at Thr495. Phosphorylation was measured by western blot using mouse mesenteric arteries that were cannulated and pressurized ( $75 \mathrm{~mm} \mathrm{Hg}$ ) and treated either by 1) $5 \mathrm{~min}$ of phenylephrine superfusion $\left(10^{-5} \mathrm{M}\right)$ (PE5), 2) 15 min of phenylephrine (PE15), 3) 15 min phenylephrine followed by acetylcholine $\left(10^{-4} \mathrm{M}\right)(\mathrm{PE}+\mathrm{ACh})$, or 4) 20 min time control with no treatment (NT) [4-5 arteries pooled per treatment per blot; 5 blots performed]. These treatments allowed correlation between vasomotor changes, namely maximal constriction (PE5), slow vasodilation (PE15), and maximal dilation (PE $+\mathrm{ACh}$ ), and relative phosphorylation changes. Phosphorylation of eNOS at Ser1179 was increased relative to NT by more than 2-fold at PE5 and remained similarly increased at PE15 and PE + ACh. Phosphorylation of eNOS at Thr495 was less in all treatments relative to NT, but not significantly. Treatment with L-NAME $\left(10^{-4} \mathrm{M}\right)$ or endothelial denudation indicated that the slow dilation in response to phenylephrine was completely due to nitric oxide synthase and was endothelial dependent. These results indicate that eNOS phosphorylation at Ser1179 occurs before the slow dilation and is not actively involved in this vasodilation or dilation to acetylcholine, but may play a permissive role in eNOS activation by other mechanisms. It is not yet known what mechanism is responsible for Ser1179 phosphorylation with phenylephrine stimulation.
\end{abstract}

(c) 2012 Elsevier Inc. All rights reserved.

\section{Introduction}

Endothelial nitric oxide synthase (eNOS), which produces the gas nitric oxide, is an important enzyme for cardiovascular health as it is essential for maintenance of normal blood pressure (Shesely et al., 1996), for preventing atherosclerosis (Kuhlencordt et al., 2001), for angiogenesis (Murohara et al., 1998; Yu et al., 2005), and for vasodilation and remodeling in large arteries (Brandes et al., 2000; Rudic et al., 1998). It is activated by shear stress, hypoxia, and endothelial agonists, such as bradykinin, estrogen, and VEGF (Sessa, 2004). Its catalytic activity is regulated by intracellular calcium, phosphorylation, dimerization, and interaction with other proteins (Rafikov et al., 2011). Phosphorylation changes occur at several sites and stimulation of cultured endothelial cells with agonists induces increased eNOS phosphorylation at Ser1179 and desphosphorylation at Thr495 (human sequence nomenclature) (Harris et al., 2001; Rafikov et al., 2011; Sessa, 2004). Phosphorylation at Ser1179 seems to be a critical event because in cultured endothelial cells, alteration of Ser1179 to alanine results in decreased production of nitric oxide (Dimmeler et al., 1999; Fulton et al., 1999; Scotland et al., 2002), and conversion to aspartate (mimicking phosphorylation) causes maximal enzymatic activity of the enzyme and independence

\footnotetext{
* Corresponding author. Tel.: +1 7572212784.

E-mail address: rlooft@wm.edu (R.C. Looft-Wilson).
}

from increases in calcium concentration (Dimmeler et al., 1999). Moreover, in intact carotid arteries, transgenic alteration of amino acid 1179 from serine to alanine or adenoviral reconstitution of eNOS knockout mice to Ala1179 impairs acetycholine-induced vasodilation (Atochin et al., 2007; Scotland et al., 2002). It is unclear, however, how and at what sites eNOS is phosphorylated in intact arteries in response to agonists and over time and how this correlates with vasomotor responses.

Generation of nitric oxide is also stimulated in some vessels during vasoconstriction by activation of sympathetic nerves (Boric et al., 1999) or by the alpha ${ }_{1}$-adrenergic receptor agonist, phenylephrine (Dora et al., 1997; Tuttle and Falcone, 2001). Dora et al. (1997) were the first to show that with stimulation of hamster arterioles with phenylephrine lead to an increase in endothelial cell calcium and subsequent generation of nitric oxide and attenuation of constriction. The rise in endothelial cell calcium provided evidence for gap junction communication from smooth muscle to endothelial cells (myoendothelial gap junction communication), because phenylephrine is not known to have direct effects on endothelial cells. This phenomenon has also been observed in rat skeletal muscle arterioles (Tuttle and Falcone, 2001) and in mouse mesenteric arteries (Nausch et al., 2012). In this latter study, it was recently shown that in mouse mesenteric arteries, smooth muscle alpha $_{1}$-adrenergic receptor stimulation with phenylephrine results in $\mathrm{IP}_{3}$-mediated release of $\mathrm{Ca}^{++}$from the endothelial sarcoplasmic reticulum, presumably due to diffusion of $\mathrm{IP}_{3}$ from smooth muscle to endothelial cells via gap junctions (Nausch et al., 2012). Others have provided 
evidence that these particular arteries have myoendothelial gap junction communication to support this conclusion (Dora et al., 2003; Saliez et al., 2008). This increase in endothelial cell calcium at the myoendothelial junction potentially stimulates eNOS, as it has recently been shown that eNOS can reside in the myoendothelial junction in mouse arteries (Straub et al., 2011). It is unknown whether phosphorylation of eNOS also occurs during this process.

In this study, we sought to determine whether phenylephrine stimulation of intact arteries results in eNOS regulation via phosphorylation. We show that in intact, cannulated mouse mesenteric arteries, phenylephrine induced eNOS Ser1179 phosphorylation within 5 min, even as the artery reached maximal constriction. As the artery partially dilated in reaction to phenylephrine-induced stimulation of NOS, there was no further increase in Ser1179 phosphorylation, nor was there any further increase when acetylcholine was added to induce maximal dilation, despite its NOS dependence. These data indicate that phosphorylation of eNOS on Ser1179 does not necessarily correspond to vasodilatory responses and full phosphorylation at this site does not result in maximal dilation, but suggests that this phosphorylation may act permissively when other mechanisms of regulation are activated.

\section{Methods}

\subsection{Measurement of in vitro artery function}

All experimental procedures were approved by the William \& Mary Institutional Animal Care and Use Committee and are in accordance with the Guide for the Care and Use of Laboratory Animals (National Research Council). Male C57BL/6 mice ( 12 weeks old) were anesthetized with sodium pentobarbital (Nembutal, $50 \mathrm{mg} / \mathrm{kg}$ body weight, intra-peritoneal), the intestines were removed and euthanasia resulted from exsanguination. The intestines along with the mesenteric arcade were placed in cold PSS-MOPS buffer (in mM: $145 \mathrm{NaCl}, 4.7 \mathrm{KCl}, 2.0$ $\mathrm{CaCl}_{2}, 1.17 \mathrm{MgSO}_{4}, 1.2 \mathrm{NaH}_{2} \mathrm{PO}_{4}, 2.0 \mathrm{MOPS}, 0.02$ EDTA, 5.0 glucose, 2.0 pyruvate, $\mathrm{pH}$ 7.4). Two first order mesenteric arteries were removed from each mouse, cannulated in separate vessel chambers (Danish Myo Technology, Aarhus, DK) filled with PSS-MOPS, secured with nylon suture (8-0, S\&T, Neuhausen, Switzerland), gently cleared of blood, and pressurized to $75 \mathrm{~mm} \mathrm{Hg}$ with PSS-MOPS $+1 \%$ bovine serum albumin perfusion, with no subsequent flow through the lumen. The chambers were mounted on stages of two separate pressure myograph microscope systems (Danish Myo Technology) and equilibrated at $37^{\circ} \mathrm{C}$ for $30 \mathrm{~min}$, with constant superfusion (superfusate not re-circulated) before testing functional responses. Luminal diameter was measured using a $10 \times$ objective, ccd camera, and VediView Software (Danish Myo Technology) on one system and Ionoptix software (Milton MA) on the other system using on-screen cursors.

After the $30 \mathrm{~min}$ equilibration, mounted vessels were tested in one of 6 different protocols: 1) no treatment (NT), which consisted of 20 min of equilibration with the superfusate re-circulated $(100 \mathrm{ml}$ total volume). This treatment acted as a time control in which no drugs were added, and diameter was measured before and after equilibration; 2) phenylephrine treatment $\left(10^{-5} \mathrm{M}\right.$ in $100 \mathrm{ml}$ superfusate) for 5 min (PE5) with diameter measured every minute; 3) phenylephrine treatment $\left(10^{-5} \mathrm{M}\right.$ in $100 \mathrm{ml}$ superfusate) for $15 \mathrm{~min}$ (PE15) with diameter measured every minute; 4$)$ acetylcholine treatment $\left(10^{-4} \mathrm{M}\right.$ in $100 \mathrm{ml}$ superfusate) for $5 \mathrm{~min}(\mathrm{PE}+\mathrm{ACh}$ ) after pre-constriction with $10^{-5} \mathrm{M}$ phenylephrine for $15 \mathrm{~min}$. Diameter was measured every minute after phenylephrine addition and subsequent acetylcholine addition. 5) Treatment with L-NAME $\left(10^{-4} \mathrm{M}\right.$, in lumen perfusate and in superfusate) beginning during cannulation, followed by treatment with phenylephrine and acetylcholine as in treatment \#4 (L-NAME), to determine how much of the constriction and dilation with these drugs was due to nitric oxide synthase/s. 6) Arteries were treated with air through the lumen for 10-15 min to destroy the endothelium, followed by treatment with phenylephrine and acetylcholine as in treatment \#4 (Denuded), to determine how much of the constriction and dilation to drugs was endothelial dependent. All arteries were frozen immediately in liquid nitrogen (and stored at $-80{ }^{\circ} \mathrm{C}$ ) after each of these treatments for western blot analysis, with the exception of the L-NAME arteries. Maximal diameter was measured in the L-NAME group after superfusion with $\mathrm{Ca}^{++}$-free PSS-MOPS $+1 \mathrm{mM}$ EGTA for at least $15 \mathrm{~min}$. In the other groups, maximal diameter was estimated by the initial diameter upon chamber mounting when the vessel was bathed in cold PSS-MOPS. Previous results in our laboratory have shown this to be a close estimate given that the vessel is fully relaxed in this state and there is no basal tone.

All groups had similar luminal diameters at the initial measurement in cold PSS (mean \pm SEM: NT: $230 \pm 5 \mu \mathrm{m}$; PE5: $235 \pm 6 \mu \mathrm{m}$; PE15: $234 \pm 6 \mu \mathrm{m}$; PE + ACh: $242 \pm 8 \mu \mathrm{m}$; L-NAME: $224 \pm 12$; Denuded: $232 \pm 11)$. In four of the groups the baseline diameter after $30 \mathrm{~min}$ of equilibration at $37{ }^{\circ} \mathrm{C}$ was either not changed (PE5: 229 $\pm 8 \mu \mathrm{m}$; PE15: $235 \pm 7 \mu \mathrm{m})$, or increased slightly but significantly $(\mathrm{p}<0.05$, paired t-test) (NT: $235 \pm 5 \mu \mathrm{m}$; PE + ACh: $245 \pm 8 \mu \mathrm{m}$ ), indicating an absence of basal tone, which is consistent with our previous results (Looft-Wilson et al., 2008). However, the L-NAME and Denuded groups had significantly decreased $(\mathrm{p}<0.05$, paired t-test) diameters after the 30 min equilibration (L-NAME: $195 \pm 11 \mu \mathrm{m}$; Denuded: $198 \pm 12 \mu \mathrm{m}$ ), indicating basal nitric oxide release from the endothelium in this artery type.

In a separate set of experiments arteries underwent one of three treatments: 1) NT, 2) PE15, or 3) treatment with phenylephrine for $15 \mathrm{~min}$ in the presence of the alpha $\mathrm{a}_{1}$-adrenergic receptor antagonist, prazosin $(500 \mathrm{nM})$ in the superfusate beginning at the 30 min equilibration (Prazosin). These experiments were performed to confirm that the constriction to phenylephrine was entirely dependent on alpha ${ }_{1}$ adrenergic receptor stimulation. These arteries had similar initial diameters (NT: 256 \pm 24 ; PE15: $238 \pm 15$; Prazosin: $241 \pm 7$ ) with no change after 30 min of equilibration (NT: $260 \pm 22$; PE15: $241 \pm 14$; Prazosin: $245 \pm 6)$.

In a preliminary set of experiments, arteries were treated with increasing doses of phenylephrine $\left(10^{-9} \mathrm{M}-10^{-4} \mathrm{M}\right)$ in a cumulative manner, with 5 min allowed after each dose for equilibration. These experiments were performed to determine the dose of phenylephrine that produces the maximal constriction, the variability with each dose, and the effective concentration to produce a 50\% constriction (EC-50). This curve indicated that a dose of $10^{-5} \mathrm{M}$ phenylephrine produced a maximal response with low variability (SEM) (Supplementary Fig. 1), so this dose of phenylephrine was chosen as the single treatment dose to assess functional responses and eNOS phosphorylation status in the experiments described above. Although this dose produces a maximal drug response, greater constriction is still possible in this artery when endothelium-dependent vasodilation is impaired either through denudation or NOS inhibition.

Vessel diameter responses were calculated by: diameter with drug/ baseline diameter. Vessel function experiments were performed in two arteries from each mouse, but different treatments were performed in each of the two arteries.

\subsection{Phosphorylation of eNOS in mesenteric arteries after drug treatment}

Western blotting was performed to quantify the relative phosphorylation of eNOS at Ser1179 and Thr495 in pooled mesenteric arteries (4-5 arteries for a given treatment per western blot). Tissues were homogenized in $80-100 \mu$ lysis buffer with phosphatase inhibitors [50 mM Tris- $\mathrm{HCl}, 100 \mathrm{mM} \mathrm{NaF}, 15 \mathrm{mM} \mathrm{Na} \mathrm{P}_{2} \mathrm{O}_{7}, 1 \mathrm{mM} \mathrm{Na} \mathrm{VO}_{4}, 1 \%$ Triton X-100, and 1:200 protease inhibitor cocktail solution (\#P2714, Sigma, St. Louis, MO); $\mathrm{pH}=7.6$ ], incubated for $1 \mathrm{~h}$ at $4{ }^{\circ} \mathrm{C}$, and centrifuged $(14,000 \mathrm{rpm}, 10 \mathrm{~min})$ to remove insoluble material. Proteins were separated by $10 \%$ SDS-PAGE (4\% stacking gel) using $25 \mu \mathrm{l}$ of protein. Proteins were transferred to a nitrocellulose membrane and total 
protein was visualized on the membrane with ponceau-S (in some but not all membranes). Membranes were cut in half between the 75 and $50 \mathrm{kDa}$ markers, and the higher molecular weight half was immunolabeled first for phosphorylated eNOS, either pSer1179-eNOS (1:750; BD Biosciences, San Jose, CA) or pThr495-eNOS (1:1000, BD Biosciences), followed by HRP-conjugated secondary antibody (anti-mouse 1:5000, Pierce Biotechnology, Rockford, IL), and visualized with enhanced chemiluminescence (Pierce Biotechnology) captured on film. The membrane was then stripped for 20 min (Restore Western Blot Stripping Buffer, Thermo Scientific, Waltham, MA), and re-probed with eNOS (1:1000, BD Biosciences) using the same procedure as with the phosphorylated forms. We have previously shown that this stripping protocol results in negligible residual labeling of the phosphorylated form even with long film exposure times (Looft-Wilson et al., 2008). The half of the membrane containing the lower molecular weight proteins was labeled for gapdh (1:1000; Millipore, Temucula, CA) or $\beta$-actin (1:1000; Cell Signaling Technology, Danvers, MA).

\subsection{Data analysis}

Statistics were performed using Prism software (GraphPad Software, Inc., San Diego, CA). Vessel responses were compared between groups by two-way ANOVA with repeated measures (Bonferroni post-hoc analysis). Protein bands were quantified by scanning the film and determining the density of each band using Image J software (NIH). Single bands were detected on the membranes at the appropriate sizes: gapdh (38 kDa), pS1179eNOS (140 kDa), pT495-eNOS (140 kDa), eNOS (140 kDa), and beta-actin (45 kDa). Phosphorylated eNOS protein was normalized to total eNOS protein and this ratio was normalized to the no treatment (No T) values in each blot to determine the relative change in eNOS phosphorylation at each site in response to the drug treatments, and to allow combining of different blots. Treatments were compared by one-way ANOVA (Bonferroni post-hoc analysis). Initial diameters between groups were compared by one-way ANOVA (Bonferroni post-hoc analysis), and comparisons of initial diameters to baseline diameters after 30 min equilibration were compared within each group by paired t-test. Significance was at $p<0.05$. The EC-50 for phenylephrine was calculated for each vessel in the phenylephrine cumulative dose-response trial using GraphPad Prism's sigmoidal plot function.

\section{Results}

Addition of phenylephrine to mesenteric arteries caused an initial constriction followed by a slow dilation over 15 min (Fig. 1A and B), similar to the responses observed in mouse thoracodorsal arteries (Straub et al., 2011). This slow dilation was dependent upon NOS activation because it was blocked by incubation with L-NAME $\left(10^{-4} \mathrm{M}\right)$, and required the presence of the endothelium because it was eliminated by denudation. Subsequent addition of acetylcholine resulted in full dilation that was $~ 50 \%$ dependent upon NOS activation as indicated by the impaired dilation after 5 min of acetylcholine addition, and fully dependent upon an intact endothelium as dilation was nearly eliminated by denudation. The early dilation after acetylcholine addition (1-2 min) is less dependent upon NOS as indicated by the near maximal dilation (relative to baseline diameter) in the presence of L-NAME at these time-points. In addition, we have previously provided evidence that this early response in these arteries is attributable to a mechanism consistent with endothelium-dependent hyperpolarization (EDH), because it is blocked by high $\mathrm{K}^{+}$and is not due prostacyclin and only partially due to NOS (Looft-Wilson et al., 2008). We also showed in this previous study that the artery will remain $\sim 50 \%$ dilated for at least 30 min after acetylcholine addition and this dilation is due to NOS, as it is completely blocked by L-NAME. When these responses were examined in terms of absolute diameter changes (Fig. 1B), it is clear that L-NAME and denudation resulted in greater basal tone and a deeper


Fig. 1. Artery diameter responses (mean $\pm \mathrm{SEM})$ to phenylephrine $\left(10^{-5} \mathrm{M}\right)$ superfusion followed by acetycholine $\left(10^{-4} \mathrm{M}\right)$ without (Control) or with L-NAME $\left(10^{-4} \mathrm{M}\right)$ (L-NAME) in the superfusate and luminal perfusate, or with denudation (Denuded). (A) Diameter responses are represented as \% of baseline diameter measured at $30 \mathrm{~min}$ post-equilibration. (B) Absolute diameter values are plotted for the same vessels as in panel A. Maximal diameters (estimated from initial diameter in cold PSS) were similar between the groups, indicating that the L-NAME and Denuded groups had basal tone after equilibration. * significantly $(\mathrm{p}<0.05)$ different from both L-NAME and Denuded. \# significantly $(\mathrm{p}<0.05)$ different from L-NAME. Twenty-two of the Control arteries are the same ones depicted in the PE + ACh group in Fig. 2 and analyzed by western blot in Figs. 3 and 4, and three of the Control arteries and three of the Denuded arteries were analyzed by western blot in Fig. 5. Values for one of the Control arteries were estimated at times 1-5 min based on group average due to a computer crash during these timepoints for this artery.

constriction, which is consistent with the results found by others (Dora et al., 2000).

Arteries in the different treatment groups had similar vasomotor responses to agonists up to the time-point at which they were frozen (Fig. 2). The corresponding eNOS phosphorylation events at the various time-points are shown in Figs. 3 and 4 (individual western blot films and quantifications are displayed in Supplementary Fig. 4 and the Supplementary table). Phosphorylation of eNOS at Ser1179 relative to total eNOS expression (Fig. 3) was significantly increased by more than 2-fold after 5 min of phenylephrine treatment, and remained similarly increased after 15 min of phenylephrine treatment and after acetylcholine addition. Even though the arteries in PE15 and PE + ACh groups were 63\% and 199\% more dilated, respectively, than PE5 (\% dilation: PE5= $30.1 \pm 9.6 \% ; \mathrm{PE} 15=49.1 \pm 12.8 \% ; \mathrm{PE}+\mathrm{ACh}=90.1 \pm 10.1 \%)$, the relative 


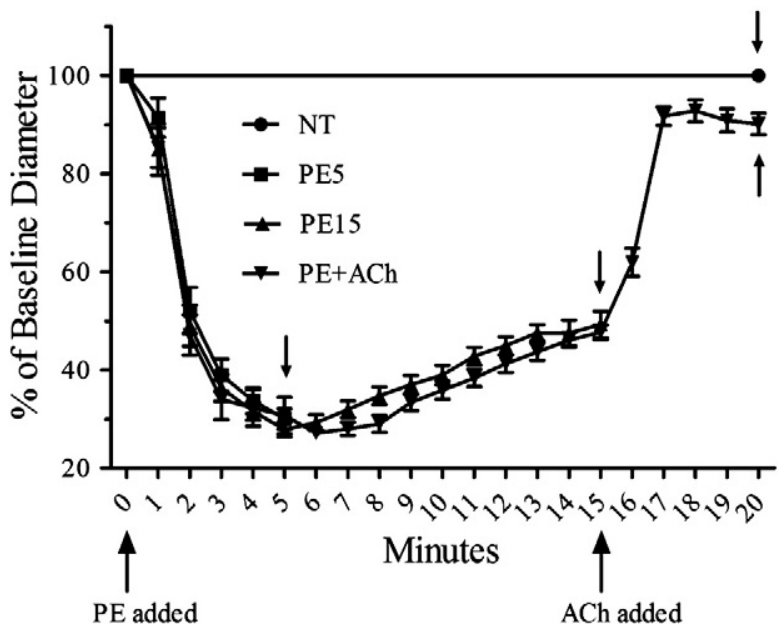

Fig. 2. Artery diameter responses for all vessels in each of the four treatments used to detect eNOS phosphorylation. Each treatment group contains 22 total arteries, with 4-5 arteries used in each of the 5 western blot trials presented in Figs. 3 and 4. Arteries were frozen immediately after the last diameter measurement in each of the four different treatments, which corresponds to $20 \mathrm{~min}$ for the NT and PE + ACh groups, 5 min for the PE5 group and 15 min for the PE15 group (small arrows show the time-point when arteries were frozen in a given group). Values for 1-5 min are missing for one vessel in the PE + ACh group due to a computer crash.

phosphorylation at Ser1179-eNOS was not different. Phosphorylation of eNOS at Thr495 (Fig. 4) showed a trend toward decreasing in all groups relative to the control group (NT), but did not reach significance. Total eNOS expression was not different between the groups when normalized to the housekeeping gene gapdh (Supplementary Fig. 3).

When eNOS expression was compared between three of the Control arteries in Fig. 1 and three of the Denuded arteries in Fig. 1, it is clear that the majority of eNOS is expressed in the endothelium because the eNOS signal in the Denuded group was only $~ 5 \%$ as strong as the signal in the Control group (Fig. 5). The residual signal in the Denuded group could mean that a minor amount of eNOS is expressed in other cell types in the vessel wall, or that denudation was not complete. Incomplete denudation is a possibility because there are many endothelial cell projections that extend through the basal lamina to the smooth muscle and eNOS has been indentified in these projections (Straub et al., 2011).

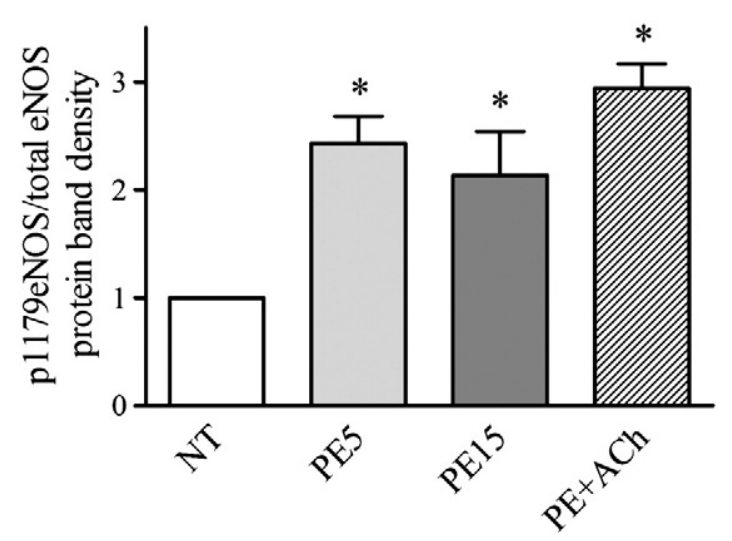

Fig. 3. eNOS phosphorylation at Ser1179 increased significantly $(\mathrm{p}<0.05)$ relative to control arteries (NT) after treatment with phenylephrine $\left(10^{-5} \mathrm{M}\right)$ for 5 (PE5) or $15 \mathrm{~min}$ (PE15), or treatment with PE for 15 min followed by 5 min with acetylcholine $\left(10^{-4} \mathrm{M}\right)$ $(\mathrm{PE}+\mathrm{ACh})$. There were no differences in phosphorylation between the drug treatment groups. Data are the mean of 5 western blots, with 4-5 arteries pooled for each treatment in a given western blot.

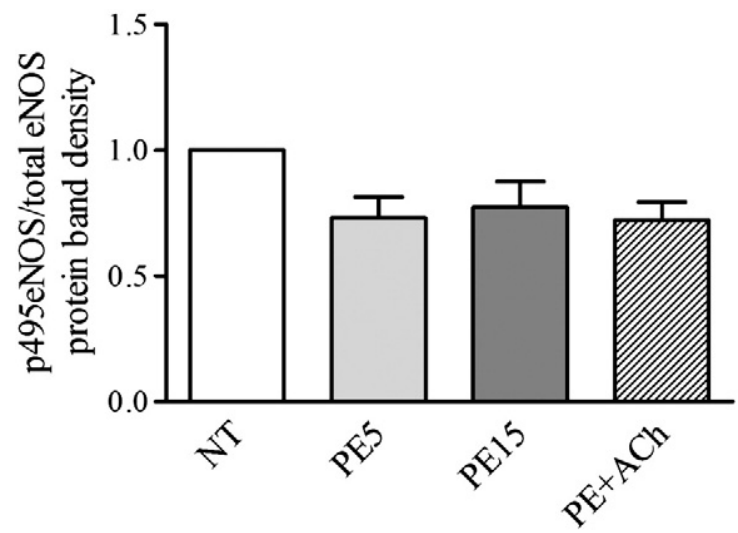

Fig. 4. eNOS phosphorylation at Thr495 was decreased, but not significantly, relative to control arteries (NT) after treatment with phenylephrine $\left(10^{-5} \mathrm{M}\right)$ for 5 (PE5) or $15 \mathrm{~min}$ (PE15), or treatment with PE for 15 min followed by 5 min with acetylcholine $\left(10^{-4} \mathrm{M}\right)$ $(\mathrm{PE}+\mathrm{ACh})$. There were no differences in phosphorylation between the drug treatment groups. Data are the mean of 5 western blots, with $4-5$ arteries pooled for each treatment in a given western blot.

\section{Discussion}

This study is the first to show the time-course of eNOS phosphorylation with alpha $_{1}$-adrenergic stimulation of smooth muscle and it revealed some unexpected results. Because stimulation of intact arteries with phenylephrine has been shown to result in both increased endothelial cell calcium and eNOS activation, it might be assumed that the increased calcium was responsible for the activation of eNOS, particularly because both the increase in calcium and expression of eNOS are found in the endothelial projections at the myoendothelial junction in mouse thoracodorsal arteries (Straub et al., 2011). It is unexpected that phosphorylation of eNOS would occur as a result of phenylephrine treatment and suggests that additional communication occurs from smooth muscle to endothelial cell either through gap junctions or possibly via a smooth muscle autacoid that activates enzymatic processes leading to phosphorylation of eNOS at Ser1179. There are several enzymes known to phosphorylate eNOS at this site, including AKT1,
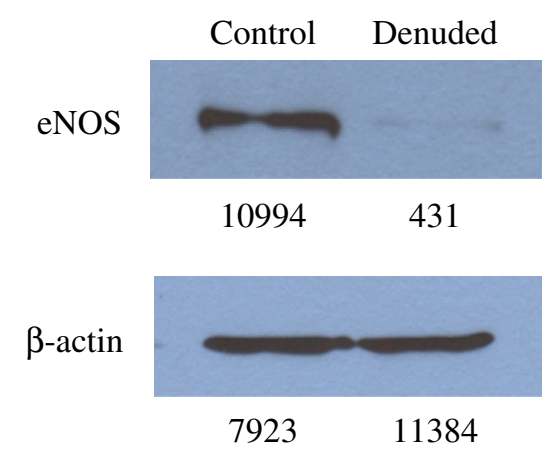

$\begin{array}{lll}\text { eNOS/ } \beta \text {-actin } & 0.831 & 0.038 \\ \text { Normalized } & 1.000 & 0.046 \\ \text { To Control } & & \end{array}$

Fig. 5. eNOS is highly expressed in Control arteries ( $\mathrm{n}=3$ pooled arteries), but relative expression declines by $\sim 95 \%$ in Denuded arteries ( $n=3$ pooled arteries), when normalized to the housekeeping gene, beta-actin. This indicates that eNOS expression is largely endothelial. The lower membrane was first probed for gapdh, but the signal was too weak, so it was stripped and re-probed for beta-actin. Integrated density values (measured with Image J) are shown below each band. The ratio of these values for eNOS/beta-actin are calculated below the films, and then normalized to the Control ratio for relative comparison between the two groups. 
AMPK, PKA, and CaMKII (Chen et al., 1999; Fleming et al., 2001; Fulton et al., 1999; Gallis et al., 1999; Harris et al., 2001; Michell et al., 2001, 1999). It is unclear which of these pathways are involved and how they are activated by alpha ${ }_{1}$-adrenergic stimulation, but CaMKII is activated by increased intracellular calcium, so this is a possible mechanism (Fleming et al., 2001).

Also, unexpectedly, the vasomotor tone of the intact vessel did not correlate with phosphorylation status of eNOS. Firstly, 5 min after phenylephrine stimulation, the artery was at its maximal point of constriction, yet eNOS was also at a level of Ser1179 phosphorylation that appears to be maximal. Blockade of nitric oxide synthases or denudation at this time-point did not increase the relative magnitude of constriction (\% constriction) indicating that the influence of eNOS on vasomotor tone at this time-point is minimal. It should be noted, however, that the absolute constriction is greater due to the increase in basal tone with NOS inhibition and denudation, as illustrated in Fig. 1B. Secondly, NOS and an intact endothelium were critical for dilation that occurred from 5 to $15 \mathrm{~min}$ after phenylephrine stimulation, and at its greatest dilation at 15 min ( $50 \%$ dilation), but there was no additional increase in eNOS phosphorylation at Ser1179. Finally, full dilation induced by acetylcholine, which was $50 \%$ NOS-dependent at 5 min after stimulation, did not result in any further Ser1179 phosphorylation. These data indicate that artery dilation induced by either phenylephrine or acetylcholine was not accompanied by active phosphorylation at Ser1179. Rather, it appears that phenylephrine stimulation induced Ser1179 phosphorylation before the functional contribution of eNOS and thus may play a permissive role in its activation by other mechanisms. For example, it may increase the sensitization to intracellular calcium as shown in cultured endothelial cells (McCabe et al., 2000). This study does not provide direct evidence, however, as to the mechanism by which eNOS is ultimately activated, which may involve one or more mechanisms.

Typically, endothelial cells stimulated by agonists have decreased phosphorylation of eNOS on Thr495 (Fleming et al., 2001; Harris et al., 2001). In this study, there was a trend for reduced Thr495 phosphorylation, but it did not reach significance. It is possible that intact arteries have lower basal levels of Thr495 phosphorylation than cultured endothelial cells, such that agonist-induced stimulation has a negligible effect at this site, or that this site is regulated differently in intact arteries compared to cultured endothelial cells.

This study utilized first order mesenteric arteries as a model for several reasons. They are large enough to examine both functional responses and to provide enough tissue (even though it has to be pooled from several arteries) for western blot analysis. It also has relatively robust reliance on eNOS for both phenylephrine-induced dilation and endothelium-dependent dilation (Fig. 1). In smaller mesenteric arteries this NOS-dependence is less important and there is more reliance on endothelium-dependent hyperpolarization (EDH) in mice (Nausch et al., 2012) and rats (Garland and McPherson, 1992; Parsons et al., 1994; Shimokawa et al., 1996). This is likely the reason why a recent study by Nausch et al. (2012) found that with phenylephrine stimulation, the resulting vasodilation was not dependent upon NOS, but rather endothelial $\mathrm{K}^{+}$channel activation consistent with EDH mechanisms. The first order mesenteric arteries do rely on mechanisms consistent with EDH for the intial dilation to acetycholine (Looft-Wilson et al., 2008), but all the slow dilation secondary to phenylephrine stimulation appears to be NOS-dependent as shown in Fig. 1.

The final reason for using this model is that examination of phenylephrine-induced responses in this vessel is of physiological importance. The mesenteric vascular bed is highly innervated by sympathetic nerves and contributes significantly to vascular resistance in rodents (Christensen and Mulvany, 1993; Long and Segal, 2009). Thus, it is likely that vasodilatory mechanisms will occur in the presence of some degree of underlying sympathetic tone. Moreover, it has been shown in mouse mesenteric arteries that the norepinephrine released from the sympathetic varicosities is the primary sympathetic neurotransmitter responsible for the slow dilation that occurs in response to sympathetic nerve stimulation, and that the alpha ${ }_{1}$ adrenergic receptors are the adrenergic receptors involved (Nausch et al., 2012). We confirmed in this study that constriction to phenylephrine is completely blocked by the alpha ${ }_{1}$-adrenergeric receptor blocker prazosin (Supplementary Fig. 2). Therefore, phenylephrine stimulation of the intact artery, as performed in this study, is a legitimate way to mimic the effects of nerve stimulation in vitro in a targeted way.

A possible limitation of using phenylephrine superfusion to mimic the effects of norepinephrine release from sympathetic nerves is that the phenylephrine likely diffuses to the endothelial cells and could interact with receptors on these cells if present. This is unlikely, however, because at least in hamster arterioles, there are no alpha-adrenergic receptors expressed in endothelial cells and these vessels respond to phenylephrine treatment in a similar way as mesenteric arteries, with an increase in endothelial intracellular calcium (Jackson et al., 2008). Moreover, in mouse mesenteric arteries it has been shown that picospritzing phenylephrine onto the endothelium (such that it does not interact with the smooth muscle) does not result in increased endothelial cell calcium (Nausch et al., 2012). Thus, it is very unlikely that the artery responses are due to phenylephrine action on endothelial cells.

\section{Conclusions}

In summary, this study shows for the first time the correlation between intact artery vasomotor tone and the time-course of eNOS phosphorylation at Ser1179 and Thr495 during alpha ${ }_{1}$-adrenergic receptor stimulation and endothelium-dependent vasodilation. The significant increase in Ser1179 phosphorylation occurred shortly after phenylephrine stimulation and remained at the same level over many minutes of phenylephrine stimulation and acteylcholine stimulation, despite changes in vasomotor tone. These data suggest that the phosphorylation at this site is not controlling the NOS-dependent effect on vasomotor tone, but may be acting permissively to enhance eNOS stimulation by other mechanisms.

\section{Disclosures}

The authors of this manuscript have no conflicts of interest to disclose.

\section{Acknowledgments}

The authors thank Dr. Brennan Harris for the helpful comments in the study design and manuscript preparation. This work was supported by NIH-1R15HL082647 and NIH-1R15HL102742 (R.C. Looft-Wilson) and The American Physiological Society's Undergraduate Summer Research Fellowship (S.E. Todd, 2009; S.M. Mutchler, 2011).

\section{Appendix A. Supplementary data}

Supplementary data to this article can be found online at http:// dx.doi.org/10.1016/j.vph.2012.09.003.

\section{References}

Atochin, D.N., Wang, A., Liu, V.W., Critchlow, J.D., Dantas, A.P., Looft-Wilson, R., Murata, T., Salomone, S., Shin, H.K., Ayata, C., Moskowitz, M.A., Michel, T., Sessa, W.C., Huang, P.L., 2007. The phosphorylation state of eNOS modulates vascular reactivity and outcome of cerebral ischemia in vivo. J. Clin. Investig. 117, 1961-1967.

Boric, M.P., Figueroa, X.F., Donoso, M.V., Paredes, A., Poblete, I., Huidobro-Toro, J.P., 1999 Rise in endothelial-derived NO after stimulation of rat perivascular sympathetic mesenteric nerves. Am. J. Physiol. 277, H1027-H1035.

Brandes, R.P., Schmitz-Winnenthal, F.H., Feletou, M., Godecke, A., Huang, P.L., Vanhoutte, P.M., Fleming, I., Busse, R., 2000. An endothelium-derived hyperpolarizing factor distinct from NO and prostacyclin is a major endothelium-dependent vasodilator in resistance vessels of wild-type and endothelial NO synthase knockout mice. Proc. Natl. Acad. Sci. U. S. A. 97, 9747-9752 
Chen, Z.P., Mitchelhill, K.I., Michell, B.J., Stapleton, D., Rodriquez-Crespo, I., Witters, L.A Power, D.A., Ortiz de Montellano, P.R., Kemp, B.E., 1999. AMP-activated protein kinase phosphorylation of endothelial NO synthase. FEBS Lett. 443, 285-289.

Christensen, K.L., Mulvany, M.J., 1993. Mesenteric arcade arteries contribute substantially to vascular resistance in conscious rats. J. Vasc. Res. 30, 73-79.

Dimmeler, S., Fleming, I., Fisslthaler, B., Hermann, C., Busse, R., Zeiher, A.M., 1999. Activation of nitric oxide synthase in endothelial cells by Akt-dependent phosphorylation. Nature 399, 601-605.

Dora, K.A., Doyle, M.P., Duling, B.R., 1997. Elevation of intracellular calcium in smooth muscle causes endothelial cell generation of NO in arterioles. Proc. Natl. Acad. Sci. U. S. A. 94, 6529-6534.

Dora, K.A., Hinton, J.M., Walker, S.D., Garland, C.J., 2000. An indirect influence of phenylephrine on the release of endothelium-derived vasodilators in rat small mesenteric artery. Br. J. Pharmacol. 129, 381-387.

Dora, K.A., Sandow, S.L., Gallagher, N.T., Takano, H., Rummery, N.M., Hill, C.E., Garland, C.J. 2003. Myoendothelial gap junctions may provide the pathway for EDHF in mouse mesenteric artery. J. Vasc. Res. 40, 480-490.

Fleming, I., Fisslthaler, B., Dimmeler, S., Kemp, B.E., Busse, R., 2001. Phosphorylation of $\operatorname{Thr}(495)$ regulates $\mathrm{Ca}\left({ }^{2+}\right) /$ calmodulin-dependent endothelial nitric oxide synthase activity. Circ. Res. 88, E68-E75.

Fulton, D., Gratton, J.P., McCabe, T.J., Fontana, J., Fujio, Y., Walsh, K., Franke, T.F., Papapetropoulos, A., Sessa, W.C., 1999. Regulation of endothelium-derived nitric oxide production by the protein kinase Akt. Nature 399, 597-601.

Gallis, B., Corthals, G.L., Goodlett, D.R., Ueba, H., Kim, F., Presnell, S.R., Figeys, D., Harrison, D.G., Berk, B.C., Aebersold, R., Corson, M.A., 1999. Identification of flow-dependent endothelial nitric oxide synthase phosphorylation sites by mass spectrometry and regulation of phosphorylation and nitric oxide production by the phosphatidylinositol 3-kinase inhibitor LY294002. J. Biol. Chem. 274, 30101-30108.

Garland, J.G., McPherson, G.A., 1992. Evidence that nitric oxide does not mediate the hyperpolarization and relaxation to acetylcholine in the rat small mesenteric artery. Br. J. Pharmacol. 105, 429-435.

Harris, M.B., Ju, H., Venema, V.J., Liang, H., Zou, R., Michell, B.J., Chen, Z.P., Kemp, B.E Venema, R.C., 2001. Reciprocal phosphorylation and regulation of endothelial nitric oxide synthase in response to bradykinin stimulation. J. Biol. Chem. 276, 16587-16591.

Jackson, W.F., Boerman, E.M., Lange, E.J., Lundback, S.S., Cohen, K.D., 2008. Smooth muscle alpha1D-adrenoceptors mediate phenylephrine-induced vasoconstriction and increases in endothelial cell $\mathrm{Ca}^{2+}$ in hamster cremaster arterioles. Br. J. Pharmacol. $155,514-524$

Kuhlencordt, P.J., Gyurko, R., Han, F., Scherrer-Crosbie, M., Aretz, T.H., Hajjar, R., Picard, M.H., Huang, P.L., 2001. Accelerated atherosclerosis, aortic aneurysm formation, and ischemic heart disease in apolipoprotein E/endothelial nitric oxide synthase double-knockout mice. Circulation 104, 448-454.

Long, J.B., Segal, S.S., 2009. Quantifying perivascular sympathetic innervation: regional differences in male C57BL/6 mice at 3 and 20 months. J. Neurosci. Methods 184 124-128.

Looft-Wilson, R.C., Ashley, B.S., Billig, J.E., Wolfert, M.R., Ambrecht, L.A., Bearden, S.E 2008. Chronic diet-induced hyperhomocysteinemia impairs eNOS regulation in mouse mesenteric arteries. Am. J. Physiol. Regul. Integr. Comp. Physiol. 295, R59-R66.

McCabe, T.J., Fulton, D., Roman, L.J., Sessa, W.C., 2000. Enhanced electron flux and reduced calmodulin dissociation may explain "calcium-independent" eNOS activation by phosphorylation. J. Biol. Chem. 275, 6123-6128.

Michell, B.J., Griffiths, J.E., Mitchelhill, K.I., Rodriquez-Crespo, I., Tiganis, T., Bozinovski, S. de Montellano, P.R., Kemp, B.E., Pearson, R.B., 1999. The Akt kinase signals directly to endothelial nitric oxide synthase. Curr. Biol. 9, 845-848.
Michell, B.J., Chen, Z.P., Tiganis, T., Stapleton, D., Katsis, F., Power, D.A., Sim, A.T., Kemp, B.E., 2001. Coordinated control of endothelial nitric oxide synthase phosphorylation by protein kinase $C$ and the cAMP-dependent protein kinase. J. Biol. Chem. 276, $17625-17628$.

Murohara, T., Asahara, T., Silver, M., Bauters, C., Masuda, H., Kalka, C., Kearney, M., Chen, D., Symes, J.F., Fishman, M.C., Huang, P.L., Isner, J.M., 1998. Nitric oxide synthase modulates angiogenesis in response to tissue ischemia. J. Clin. Investig. 101, 2567-2578.

Nausch, LW, Bonev, A.D, Heppner, TJ. Tallini, Y, Kotlikoff, M. L, Nelson, MT, 2012. Sympathetic nerve stimulation induces local endothelial $\mathrm{Ca}^{2+}$ signals to oppose vasoconstriction of mouse mesenteric arteries. Am. J. Physiol. Heart Circ. Physiol. 302, H594-H602.

Parsons, S.J., Hill, A., Waldron, G.J., Plane, F., Garland, C.J., 1994. The relative importance of nitric oxide and nitric oxide-independent mechanisms in acetylcholine-evoked dilatation of the rat mesenteric bed. Br. J. Pharmacol. 113, 1275-1280.

Rafikov, R., Fonseca, F.V., Kumar, S, Pardo, D. Darragh, C. Elms, S., Fulton, D., Black, S.M. 2011. eNOS activation and NO function: structural motifs responsible for the posttranslational control of endothelial nitric oxide synthase activity. J. Endocrinol. 210, 271-284.

Rudic, R.D., Shesely, E.G., Maeda, N., Smithies, O., Segal, S.S., Sessa, W.C., 1998. Direct evidence for the importance of endothelium-derived nitric oxide in vascular remodeling. J. Clin. Investig. 101, 731-736.

Saliez, J., Bouzin, C., Rath, G., Ghisdal, P., Desjardins, F., Rezzani, R., Rodella, L.F., Vriens, J., Nilius, B., Feron, O., Balligand, J.L., Dessy, C., 2008. Role of caveolar compartmentation in endothelium-derived hyperpolarizing factor-mediated relaxation: $\mathrm{Ca}^{2+}$ signals and gap junction function are regulated by caveolin in endothelial cells. Circulation $117,1065-1074$.

Scotland, R.S., Morales-Ruiz, M., Chen, Y., Yu, J., Rudic, R.D., Fulton, D., Gratton, J.P., Sessa, W.C., 2002. Functional reconstitution of endothelial nitric oxide synthase reveals the importance of serine 1179 in endothelium-dependent vasomotion. Circ. Res. 90, 904-910.

Sessa, W.C., 2004. eNOS at a glance. J. Cell Sci. 117, 2427-2429.

Shesely, E.G., Maeda, N., Kim, H.S., Desai, K.M., Krege, J.H., Laubach, V.E., Sherman, P.A., Sessa, W.C., Smithies, O., 1996. Elevated blood pressures in mice lacking endothelial nitric oxide synthase. Proc. Natl. Acad. Sci. U. S. A. 93, 13176-13181.

Shimokawa, H., Yasutake, H., Fujii, K., Owada, M.K., Nakaike, R., Fukumoto, Y., Takayanagi, T. Nagao, T., Egashira, K, Fujishima, M. Takeshita, A 1996. The importance of the hyperpolarizing mechanism increases as the vessel size decreases in endotheliumdependent relaxations in rat mesenteric circulation. J. Cardiovasc. Pharmacol. 28, 703-711.

Straub, A.C., Billaud, M., Johnstone, S.R., Best, A.K., Yemen, S., Dwyer, S.T., Looft-Wilson, R., Lysiak, J.J., Gaston, B., Palmer, L., Isakson, B.E., 2011. Compartmentalized connexin 43 s-nitrosylation/denitrosylation regulates heterocellular communication in the vessel wall. Arterioscler. Thromb. Vasc. Biol. 31, 399-407.

Tuttle, J.L., Falcone, J.C., 2001. Nitric oxide release during alpha1-adrenoceptor-mediated constriction of arterioles. Am. J. Physiol. Heart Circ. Physiol. 281, H873-H881.

Yu, J., deMuinck, E.D., Zhuang, Z., Drinane, M., Kauser, K., Rubanyi, G.M., Qian, H.S., Murata, T., Escalante, B., Sessa, W.C., 2005. Endothelial nitric oxide synthase is critical for ischemic remodeling. Mural cell recruitment, and blood flow reserve. Proc. Natl. Acad. Sci. U. S. A. 102, 10999-11004 